\title{
Strong Local-Field Enhancement of the Nonlinear Soft-Mode Response in a Molecular Crystal
}

\author{
Giulia Folpini, Klaus Reimann, Michael Woerner, ${ }^{*}$ and Thomas Elsaesser \\ Max-Born-Institut für Nichtlineare Optik und Kurzzeitspektroskopie, 12489 Berlin, Germany \\ Johannes Hoja and Alexandre Tkatchenko \\ Physics and Materials Science Research Unit, University of Luxembourg, L-1511 Luxembourg, Luxembourg \\ and Fritz-Haber-Institut der Max-Planck-Gesellschaft, Faradayweg 4-6, 14195 Berlin, Germany
}

(Received 21 December 2016; published 1 September 2017)

\begin{abstract}
The nonlinear response of soft-mode excitations in polycrystalline acetylsalicylic acid (aspirin) is studied with two-dimensional terahertz spectroscopy. We demonstrate that the correlation of $\mathrm{CH}_{3}$ rotational modes with collective oscillations of $\pi$ electrons drives the system into the nonperturbative regime of lightmatter interaction, even for a moderate strength of the THz driving field on the order of $50 \mathrm{kV} / \mathrm{cm}$. Nonlinear absorption around $1.1 \mathrm{THz}$ leads to a blueshifted coherent emission at $1.7 \mathrm{THz}$, revealing the dynamic breakup of the strong electron-phonon correlations. The observed behavior is reproduced by theoretical calculations including dynamic local-field correlations.
\end{abstract}

DOI: 10.1103/PhysRevLett.119.097404

Soft modes are a particular type of polar low-frequency lattice vibrations in crystals. They display pronounced frequency downshifts when the material approaches a structural phase transition such as in displacive ferroelectricity. The strong coupling between electronic interband and vibrational transition dipoles can strongly enhance the vibrational oscillator strength, leading to the so-called polarization catastrophe in a phase transition from the para- to the ferroelectric state of a crystal [1]. A basic theoretical approach to describe such phenomena is the concept of local-field corrections (LFC) due to the Lorentz field determined by the macroscopic polarization of all contributing (transition) dipoles [2-5].

To illustrate this concept, we consider a cubic molecular crystal having an electronic and a vibrational oscillator on the two simple cubic sublattices [Fig. 1(a)]. The two oscillators experience the same Lorentz field $L P$ [Fig. 1(b)], leading to an increasing dipole-dipole interaction between the two sets of oscillators upon decreasing the crystal's lattice constant $a$. In the linear optical response, this results in both a Lorentz-field induced redshift of the two oscillators and a significant transfer of electronic oscillator strength to the vibrations. The resulting hybrid mode, i.e., the soft-mode, is connected with electronic polarizations and currents during its oscillation period, a fact that has been shown most directly in recent femtosecond $x$-ray diffraction experiments [6]. Parallel to the increase of vibrational oscillator strength, one expects an enhancement of its optical nonlinearity compared to uncoupled oscillators [7].

The local field $E_{\mathrm{loc}}$ in the presence of the Lorentz field is given by $E_{\mathrm{loc}}=E+L P$, where $E$ represents the external field, $L$ the Lorentz factor, and $P$ the polarization of the material [Fig. 1(b)]. In a molecular crystal, both the electronic and vibrational dipoles contribute to $P$. For strong dipole-dipole coupling, the $L P$ term can be even larger than the externally applied field $E$, having two major consequences: (i) The time structure of $E_{\mathrm{loc}}(t)$ is affected by the polarization kinetics $P(t)$, following, e.g., a freeinduction decay (FID) with a finite decoherence time; (ii) a nonlinear saturation of $P(t)$ for strong external fields $E(t)$ results in a decrease of the local field, inducing a shift of the vibrational resonance to higher frequencies.

While nonlinear local-field effects have been studied in detail for Wannier excitons in semiconductors [2-5,8-11] and collective effects in atomic vapors [12,13], the nonlinear response of soft modes to a resonant external driving field and the related LFC have remained mainly unexplored. In this Letter, we report the first study of soft-mode nonlinearities in a molecular crystal, the prototype material acetylsalicylic acid (aspirin). Applying nonlinear twodimensional terahertz (2D-THz) spectroscopy, we discern different contributions to the nonlinear response, in particular, a blueshift of the soft-mode transition frequency by more than $50 \%$ for moderate external electric field amplitudes of $E_{\mathrm{pu}} \approx 50 \mathrm{kV} / \mathrm{cm}$. Theoretical model calculations describing the nonlinear dynamics of strongly coupled electronic and vibrational two-level oscillators show that the nonlinear response is in the nonperturbative regime and exhibits the character of a soft mode close to the polarization catastrophe.

Crystals of the organic compound acetylsalicylic acid $\left[\mathrm{C}_{9} \mathrm{H}_{8} \mathrm{O}_{4}\right.$, aspirin, Fig. 1(c)] are promising candidates for studying soft-mode nonlinearities. Recent theoretical work [14] on aspirin's electronic and phononic structure has shown that vibrational modes in the $\mathrm{THz}$ frequency range exhibit a strong coupling to electron density fluctuations in the solid. The free $\mathrm{CH}_{3}$ rotation forms a strongly redshifted hybrid mode with charge oscillations of $\pi$ electrons around $1.1 \mathrm{THz}$. 


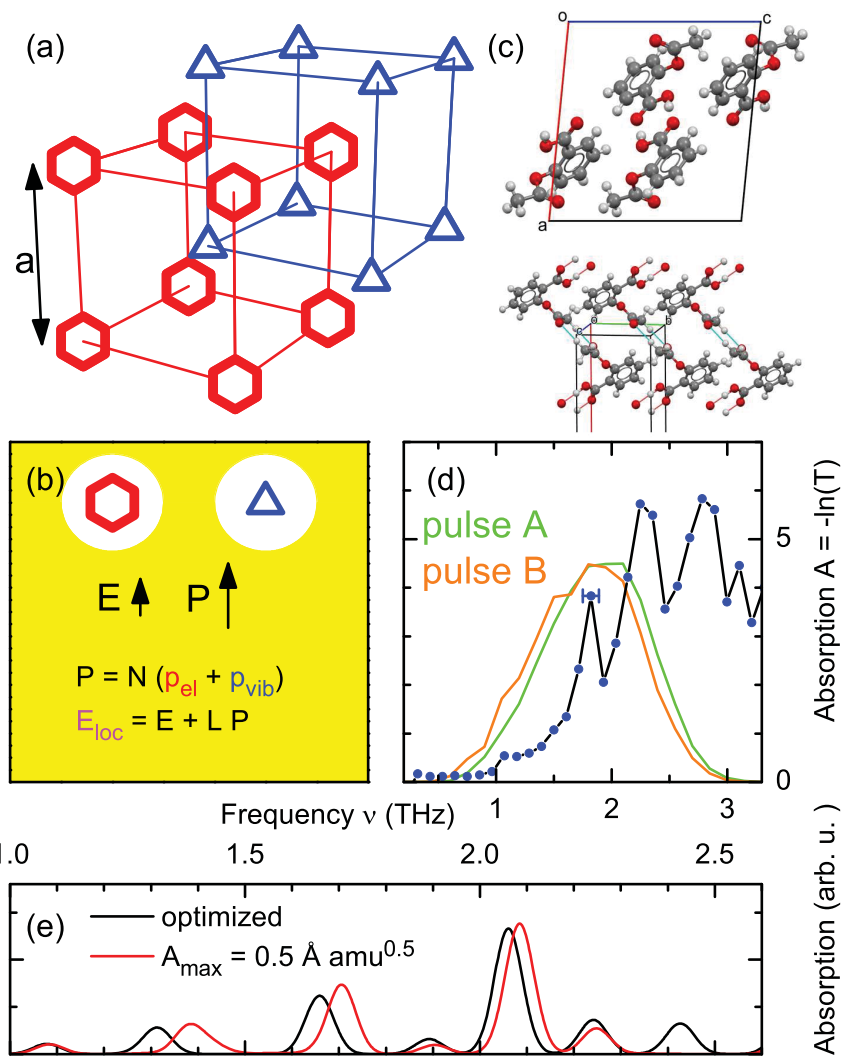

FIG. 1. (a) Schematic molecular crystal with $\mathrm{CsCl}$ structure. Electronic oscillators (represented by red benzene rings) and vibrational oscillators (represented by blue methyl rotors) are on the two simple cubic sublattices. (b) Dielectric continuum model including LFC at the spatial positions of the oscillators. Both the benzene rings and the methyl rotors have cubic environments and are placed in the center of empty spheres. The macroscopic polarization $P$ (yellow continuum) is determined by the density of electronic and vibrational oscillators. (c) Unit cell and crystal structure of aspirin. (d) Linear absorption of the aspirin sample at $T=80 \mathrm{~K}$ together with the amplitude spectra of pulses $A$ (green) and $B$ (orange). (e) First-principles calculation of the $\mathrm{THz}$ response of aspirin: Far-infrared spectrum of the optimized structure (black) and a modulated structure (red).

For the experiments, crystalline aspirin (Sigma-Aldrich, purity $>99 \%$ ) was crushed in a mortar to generate a fine powder, which was then pressed mechanically into polycrystalline layers (thicknesses from 100 to $1500 \mu \mathrm{m}$ ). The linear absorption of a 700- $\mu \mathrm{m}$ thick aspirin sample in vacuum was measured at $T=80 \mathrm{~K}$ using weak $\mathrm{THz}$ pulses in the frequency range from 0.5 up to $3 \mathrm{THz}$ [Fig. 1(d)]. The main absorption peaks appear at $1.8 \pm 0.05,2.3 \pm 0.05$, and $2.8 \pm 0.05 \mathrm{THz}$, in agreement with the literature [15]. Besides such strong peaks one clearly discerns a feature at $1.1 \pm 0.05 \mathrm{THz}$, which has been attributed to a hybrid electronic-rotational mode involving aspirin's methyl group $[14,16]$.

Nonlinear 2D-THz spectroscopy is implemented by generating two phase-locked THz pulses $A$ and $B$ separated by the coherence time $\tau$. They interact with the sample in a collinear geometry. The $\mathrm{THz}$ field transmitted through the sample held at a temperature of $80 \mathrm{~K}$ is detected in amplitude and phase as a function of real time $t$ by freespace electro-optic sampling. The field $E_{N L}(t, \tau)$ emitted by the nonlinear polarization of the sample is extracted as $E_{N L}(t, \tau)=E_{A B}(t, \tau)-E_{A}(t, \tau)-E_{B}(t)$ where $E_{A B}(t, \tau)$ is the transmitted field with both pulses $A$ and $B$ interacting with the sample, $E_{A}\left(E_{B}\right)$ being the transmitted field measured with only pulse $A(B)$ [17-21]. The two THz pulses are generated by optical rectification of $25 \mathrm{fs}$ pulses at $800 \mathrm{~nm}$ in two GaSe crystals (200 and $300 \mu \mathrm{m}$ thickness). Electro-optic sampling [22,23] in a ZnTe crystal $(100 \mu \mathrm{m})$ uses a small fraction of the Ti:sapphire amplified pulses as a probe. Spectra of the THz pulses are shown in Fig. 1(d). The peak electric fields of pulses $A$ and $B$ are 25 and $50 \mathrm{kV} / \mathrm{cm}$.

Figure 2(a) shows the electric field transmitted through the sample when both pulses $A$ and $B$ are present, Fig. 2(b) shows the nonlinearly emitted field $E_{N L}(t, \tau)$. A 2D Fourier transform of $E_{N L}(t, \tau)$ allows for separating the different

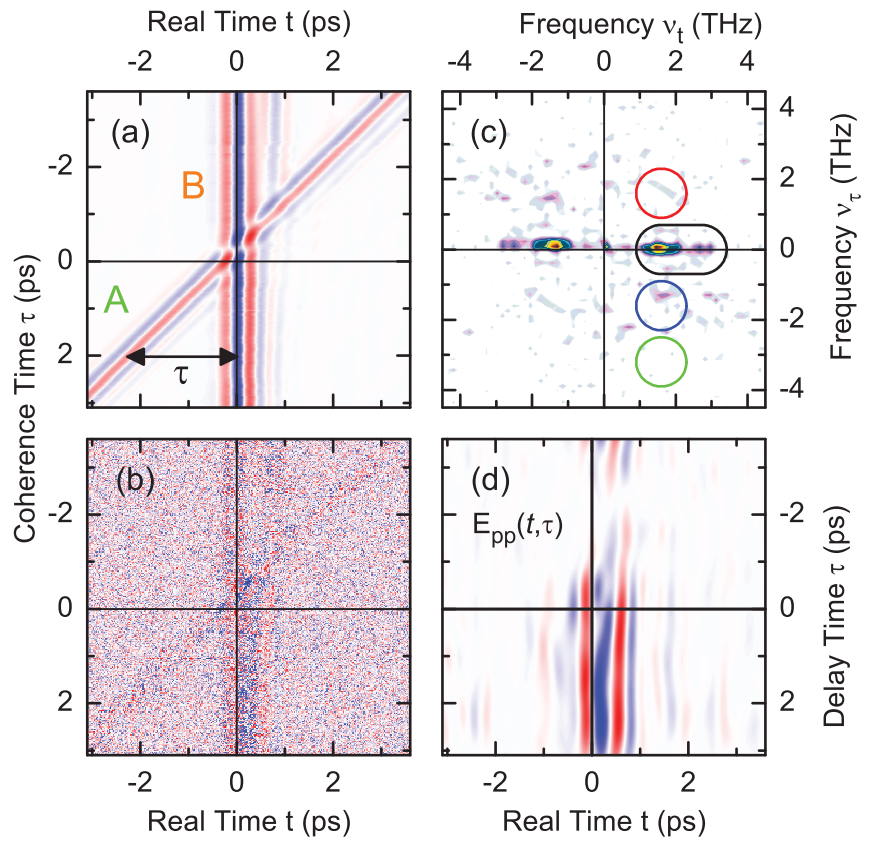

FIG. 2. (a) Contour plot of THz pulses $A$ and $B$ simultaneously transmitted through the aspirin sample held at $T=80 \mathrm{~K}$. The electric field is plotted as a function of both the real time $t$ and the delay time $\tau$ between the pulses (amplitude scale: $\pm 75 \mathrm{kV} / \mathrm{cm}$ ). (b) Electric field $E_{N L}(t, \tau)$ emitted by the nonlinear polarization of the soft mode of aspirin (amplitude scale: $\pm 1.0 \mathrm{kV} / \mathrm{cm}$ ). (c) Contour plot of the amplitude $\left|E_{N L}\left(\nu_{t}, \nu_{\tau}\right)\right|$, the 2D Fourier transform of $E_{N L}(t, \tau)$. The colored ovals indicate the position of relevant signals in the $2 \mathrm{D}$ frequency space. (d) Contour plot of the $A$-pump- $B$-probe nonlinear signal $E_{p p}^{A B}(t, \tau)$ (amplitude scale: $\pm 150 \mathrm{~V} / \mathrm{cm})$ as gained by a Fourier back transform of the $2 \mathrm{D}$ spectrum filtered by a Gaussian envelope centered on the black oval in (c). 
nonlinear contributions in the frequency domain as a function of detection frequency $\nu_{t}$ and excitation frequency $\nu_{\tau}$ [Fig. 2(c), marked by ovals]. The different components of the 2D spectrum are analyzed with the help of 2D frequency vectors [17]. The most prominent feature is the A-pump$B$-probe signal $S_{p p}^{A B}$ at $\left(\nu_{t}, \nu_{\tau}\right)=(1.5,0) \mathrm{THz}$ (black oval). Less pronounced are the rephasing $A-B-B$ photon-echo signal $S_{\mathrm{PE}}$ at $(1.5,1.5) \mathrm{THz}$ (red circle) and the $B$-pump$A$-probe signal $S_{p p}^{B A}$ at $(1.5,-1.5) \mathrm{THz}$ (blue circle). Because of the smaller electric field amplitude of pulse $A$ $\left(E_{A} \approx 0.5 E_{B}\right)$ the $B-A-A$ photon echo expected at $(1.5,-3) \mathrm{THz}$ (green circle) cannot be discerned from noise.

For a detailed analysis, we apply a 2D Gaussian spectral filter on the different signals in Fig. 2(c) and perform a Fourier back transform to the $(t, \tau)$ space. A contour plot of the $A$-pump- $B$-probe signal $E_{p p}^{A B}(t, \tau)$ [black oval in Fig. 2(c)] is shown in Fig. 2(d). This signal displays both a phase shift and a longer optical period compared to the electric field $E_{B}(t)$ of pulse $B$, which is shown in Fig. 2(a) and which is centered at a higher frequency of $2 \mathrm{THz}$ [cf., Fig. 1(d)]. A Fourier transform of the signal field $E_{p p}^{A B}(t, \tau)$ along the detection time $t$ gives the spectrally resolved pump-probe signal $S_{p p}^{A B}\left(\nu_{t}, \tau\right)=2 \operatorname{Re}\left[E_{p p}^{A B}\left(\nu_{t}, \tau\right) E_{B}^{*}\left(\nu_{t}\right)\right] /$ $\left|E_{B}\left(\nu_{t}\right)\right|^{2}$, which is plotted in Fig. 3(a) for a pump-probe



FIG. 3. (a) Spectrally resolved $A$-pump- $B$-probe signal $S_{p p}^{A B}\left(\nu_{t}, \tau\right)$ (symbols) at a delay time of $1.6 \mathrm{ps}$ [indicated by the red horizontal line in (b)]. The dashed line gives the calculated field-induced response dominated by the soft mode, the solid line the minor contributions of the modes at 1.8 and $2.3 \mathrm{THz}$. (b) Contour plot of $S_{p p}^{A B}\left(\nu_{t}, \tau\right)$ [black oval in Fig. 2(c)]. (c) Theoretical spectrally resolved pump-probe signals for a delay time of $1.6 \mathrm{ps}$ calculated for different $\mathrm{THz}$ field strengths (from left to right 30, 45 (red line), 60, and $75 \mathrm{kV} / \mathrm{cm}$ ) acting on the softmode transition. (d) Calculated pump-probe spectra as a function of delay time for a fixed $\mathrm{THz}$ field amplitude of $45 \mathrm{kV} / \mathrm{cm}$. delay of $1.6 \mathrm{ps}$ (red solid line). The spectrally resolved transmission clearly displays a spectral blueshift from $1 \mathrm{THz}$ (bleaching, $\Delta T>0$ ) to $1.5 \mathrm{THz}$ (induced absorption, $\Delta T<0)$. A contour plot of $S_{p p}^{A B}\left(\nu_{t}, \tau\right)$ along the entire delay range is shown in Fig. 3(b) pointing to a long-lived blueshift of the soft mode induced by pulse $B$.

The pump-probe signal $S_{p p}^{B A}\left(\nu_{t}, \tau\right)$ measured with the inverted pulse sequence, $B$ pump $A$ probe, is discussed in the Supplemental Material [24]. In this case, the pump pulse $B$ has a higher electric field amplitude, resulting in a distinctly different shape of $S_{p p}^{B A}\left(\nu_{t}, \tau\right)$. This behavior is in striking contrast to pump-probe experiments in the thirdorder $\left(\chi^{(3)}\right)$ limit that display different amplitudes but identical spectral shapes for the $A-B$ and the $B-A$ pulse sequences.

In the pump-probe sequences, the respective pump pulse induces both a population change and a nonlinear polarization on the vibrational transitions. The first mechanism causes a saturation contribution to the pump-probe signal that-in the $\chi^{(3)}$ limit of light-matter interaction-results in a transmission increase proportional to the fourth power of the transition dipoles interacting with the pump, i.e., on all bands overlapping with the pump spectrum [cf., Fig. 1(d)]. The experimental pump-probe spectrum in Figs. 3(a) and 3(b), however, shows a transmission decrease in the frequency range above $1.4 \mathrm{THz}$ that includes the strong bands with maxima at $1.8,2.3$, and $2.8 \mathrm{THz}$. This fact demonstrates that population saturation makes a minor contribution to the overall nonlinear response and that the nonlinear response is well beyond the perturbative $\chi^{(3)}$ limit.

The nonlinear response is governed by the nonlinear polarizations the $\mathrm{THz}$ pump (and probe) fields induce on the soft-mode transition, initially located at $1.1 \mathrm{THz}$. This mode has been shown to couple strongly to the electronic system of aspirin, mainly mediated by van der Waals interactions [14]. The interaction of the soft mode with the $\mathrm{THz}$ field is in the nonperturbative regime, leading to a pronounced nonlinear blueshift of the transition by up to $0.6 \mathrm{THz}$, i.e., more than $50 \%$ of the transition frequency. As a result, the pump-probe spectra display a transmission increase in the range below and a pronounced transmission decrease above $1.4 \mathrm{THz}$, the latter caused by the blueshifted soft-mode absorption. In our random polycrystalline sample, the $\mathrm{THz}$ field acting on a particular crystallite depends on its orientation. Different crystallites thus display different blueshifts of their soft mode [Fig. 3(c)], leading to a substantial broadening of the ensemble-averaged pumpprobe spectra. Compared to the soft mode, the vibrational modes at 1.8 and $2.3 \mathrm{THz}$ make minor contributions to the overall nonlinear response, leading to a slight spectral modulation of the transmission decrease [Fig. 3(a)].

The time evolution of the photon-echo signals reveals noninstantaneous signatures, pointing to strong contributions of the material's polarization to the total field acting 
on the soft mode. The overall behavior is close to that of a dense system of "two-level atoms" subject to a total field with a LFC contribution [2-5]. In equilibrium, the Lorentz field induces a redshift of the transition frequency compared to an isolated noninteracting oscillator, giving rise to the absorption at $1.1 \mathrm{THz}$. Any saturation of the two-level systems reduces the polarization amplitude and in turn its Lorentz field, resulting in a blueshift of the transition frequency.

The spectral envelope of the signal in Fig. 4(b) has an elliptical shape with its long axis perpendicular to the diagonal in the 2D frequency space, a behavior strikingly different from a perturbative light-matter interaction in the $\chi^{(3)}$ limit [32] (in this limit the long axis would be along the diagonal). Moreover, the rephasing photon-echo signal $E_{\mathrm{PE}}(t, \tau)$ shown in Fig. 4(a) has a finite amplitude at negative coherence times $\tau<0$. This is a hallmark of a temporally extended component in the total driving field, i.e., a contribution from the polarization term in the local field $E_{\mathrm{loc}}(t)=E(t)+L P(t)$. For strong dipole-dipole coupling, $E_{\mathrm{loc}}(t)$ is dominated by the Lorentz field having the time structure of a polarization free-induction decay $P_{\mathrm{FID}}(t)$. The extended time structure allows for additional Liouville pathways in strongly interacting media [4] with rephasing signals at $\tau<0$ (Sec. III in [24]). We conclude that the present photon-echo response is in the limit of strongly interacting media $[3,4]$.
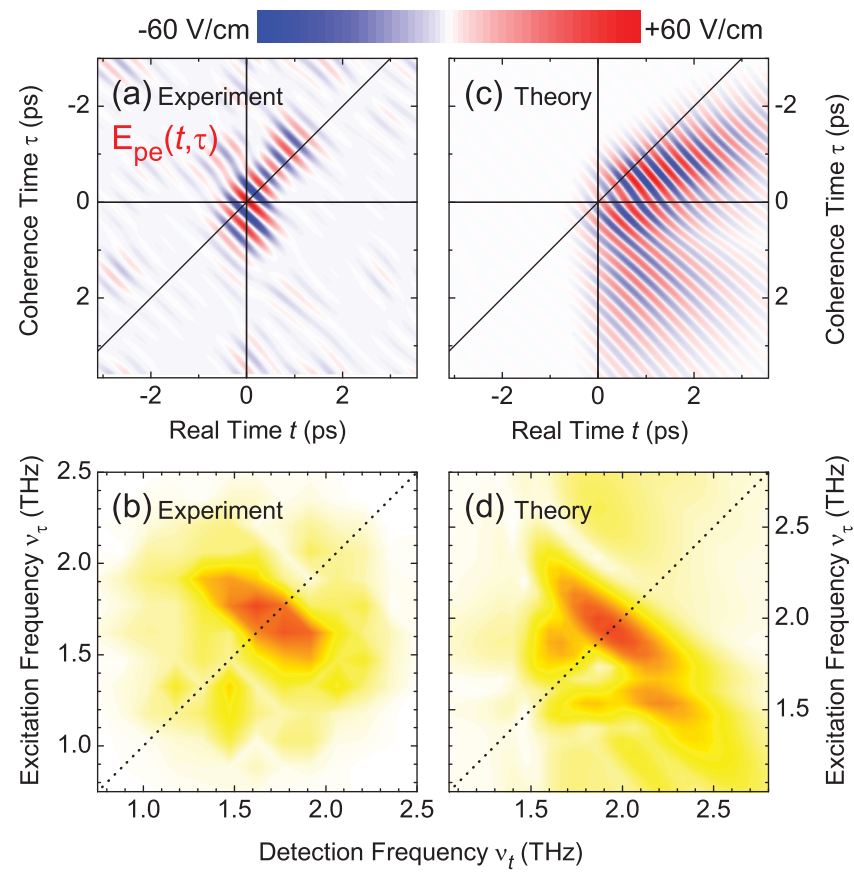

FIG. 4. (a) Contour plot of the rephasing $A-B-B$ photon-echo signal $E_{\mathrm{PE}}(t, \tau)$ as obtained by a Fourier back transform of the nonlinear signal $\left|E_{N L}\left(\nu_{t}, \nu_{\tau}\right)\right|$ within the red circle of Fig. 2(c). An enlarged view of the latter is shown in (b). [(c) and (d)] Calculated $E_{\mathrm{PE}}(t, \tau)$ and $\left|E_{\mathrm{PE}}\left(\nu_{t}, \nu_{\tau}\right)\right|$ next to their experimental counterparts in (a) and (b).
To support this interpretation of the experimental results, we performed two types of theoretical calculations described in detail in [24]. (i) We calculated the nonlinear response of two ensembles of two-level systems, located on two simple cubic sublattices [cf., Fig. 1(a)] and coupled by the local Lorentz field [Fig. 1(b)]. (ii) To get quantitative information, we performed first-principles calculations of the $\mathrm{THz}$ response for the aspirin I crystal structure [Fig. 1(c)].

In Figs. 3(c), 3(d), 4(c), and 4(d) we show results of the theoretical model (i), which are in good agreement with the experimental results in Figs. 3(a), 3(b), 4(a), and 4(b), respectively. In our calculations we varied the amplitude of the THz pulses $\left(E_{A}=0.5 E_{B}\right)$ from the $\chi^{(3)}$ limit at $E_{B}=$ $10 \mathrm{kV} / \mathrm{cm}$ over 20,40 , to $60 \mathrm{kV} / \mathrm{cm}$, i.e., into the nonperturbative regime. The calculated blueshift is proportional to the squared electric field amplitude of the $\mathrm{THz}$ pulses. The model reproduces the different shapes of the spectrally resolved pump-probe signal observed for the $A-B$ and $B-A$ pulse sequences, a clear sign of the nonperturbative light-matter interaction beyond the $\chi^{(3)}$ limit. Another indication for the nonperturbative regime is the elliptical shape of the rephasing signal $\left|E_{\mathrm{PE}}\left(\nu_{t}, \nu_{\tau}\right)\right|$ [Fig. 4(d)] with its long axis perpendicular to the diagonal in the 2D frequency space. In the time domain [Fig. 4(c)], our model reproduces the noninstantaneous response with a pronounced photon-echo signal at negative coherence times $E_{\mathrm{PE}}(t, \tau<0)$.

Our first-principles calculations [model (ii)] provide farinfrared spectra of aspirin I calculated with the PerdewBurke-Ernzerhof density functional [33] combined with the many-body dispersion method for van der Waals interactions $[34,35]$. We have calculated the $\mathrm{THz}$ spectra for both the optimized structure [black line in Fig. 1(e)] and for modulated structures, for which the atomic positions of the optimized structure have been displaced along a superposition of phonon modes corresponding to the average of the amplitude spectra of pulses $A$ and $B$ shown in Fig. 1(d) (for more details see [24]). The calculated far-infrared spectrum of the optimized structure agrees well with the experimental $\mathrm{THz}$ spectrum with deviations amounting to only about $0.2 \mathrm{THz}$. Small deviations from the experimental frequencies are expected since no thermal expansion was considered for the structure and the phonon calculations do not include any anharmonic effects. Note that the peaks located at 1.1 and $1.3 \mathrm{THz}$ in the calculated spectrum both contribute to the experimentally observed peak located at $1.1 \mathrm{THz}$. The red curve in Fig. 1(e) shows the calculated $\mathrm{THz}$ absorption for a phonon displacement amplitude of $A_{\max }=0.5 \AA \sqrt{\mathrm{amu}}$. In excellent agreement with the experiment [Fig. 3(d)] the mode centered originally at $1.3 \mathrm{THz}$ experiences the largest blueshift upon the excited phonon displacements. Indeed, this mode involves collective $\mathrm{CH}_{3}$ rotations coupled with charge density oscillations in the solid, providing support to the simplified 
model (i). The magnitude of these collective $\mathrm{CH}_{3}$ motions gets drastically reduced with increasing modulation. In the experiment [Fig. 3(b)] we see weak signatures of the theoretically predicted smaller blueshifts of the other modes [Fig. 1(e)], but because of the small signal-to-noise ratio we do not consider these further.

In conclusion, the nonlinear soft-mode response in the prototype molecular crystal aspirin is dominated by localfield effects originating from the coupling of vibrational and electronic degrees of freedom. Two-dimensional THz spectroscopy allows for separating distinct features caused by nonperturbative light-matter interactions such as spectral shifts of transition frequencies and noninstantaneous polarization contributions to the local field. Our work paves the way for future, even more detailed insight into nonlinear soft-mode dynamics and its role in light-driven structural phase transitions.

We acknowledge financial support by the Deutsche Forschungsgemeinschaft Grants No. RE 806/9-1 and No. SFB-951/A10 (J. H. and A. T.)

*woerner@mbi-berlin.de †alexandre.tkatchenko@uni.lu

[1] W. Cochran, Crystal stability and the theory of ferroelectricity, Adv. Phys. 9, 387 (1960).

[2] J. Knoester and S. Mukamel, Nonlinear optics using the multipolar Hamiltonian: The Bloch-Maxwell equations and local fields, Phys. Rev. A 39, 1899 (1989).

[3] M. Wegener, D. S. Chemla, S. Schmitt-Rink, and W. Schäfer, Line shape of time-resolved four-wave mixing, Phys. Rev. A 42, 5675 (1990).

[4] S. Schmitt-Rink, S. Mukamel, K. Leo, J. Shah, and D. S. Chemla, Stochastic theory of time-resolved four-wave mixing in interacting media, Phys. Rev. A 44, 2124 (1991).

[5] S. Mukamel, Principles of Nonlinear Optical Spectroscopy (Oxford University Press, New York, 1995).

[6] F. Zamponi, P. Rothhardt, J. Stingl, M. Woerner, and T. Elsaesser, Ultrafast large-amplitude relocation of electronic charge in ionic crystals, Proc. Natl. Acad. Sci. U.S.A. 109, 5207 (2012).

[7] I. Katayama, H. Aoki, J. Takeda, H. Shimosato, M. Ashida, R. Kinjo, I. Kawayama, M. Tonouchi, M. Nagai, and K. Tanaka, Ferroelectric Soft Mode in a $\mathrm{SrTiO}_{3}$ Thin Film Impulsively Driven to the Anharmonic Regime using Intense Picosecond Terahertz Pulses, Phys. Rev. Lett. 108, 097401 (2012).

[8] N. Peyghambarian, H. M. Gibbs, J. L. Jewell, A. Antonetti, A. Migus, D. Hulin, and A. Mysyrowicz, Blue Shift of the Exciton Resonance due to Exciton-Exciton Interactions in a Multiple-Quantum-Well Structure, Phys. Rev. Lett. 53, 2433 (1984).

[9] J. B. Stark, W. H. Knox, D. S. Chemla, W. Schäfer, S. Schmitt-Rink, and C. Stafford, Femtosecond Dynamics of Excitons under Extreme Magnetic Confinement, Phys. Rev. Lett. 65, 3033 (1990).
[10] K. W. Stone, K. Gundogdu, D. B. Turner, X. Li, S. T. Cundiff, and K. A. Nelson, Two-quantum 2D FT electronic spectroscopy of biexcitons in GaAs quantum wells, Science 324, 1169 (2009).

[11] K. W. Stone, D. B. Turner, K. Gundogdu, S. T. Cundiff, and K. A. Nelson, Exciton-exciton correlations revealed by two-quantum, two-dimensional Fourier transform optical spectroscopy, Acc. Chem. Res. 42, 1452 (2009).

[12] F. Gao, S. T. Cundiff, and H. Li, Probing dipole-dipole interaction in a rubidium gas via double-quantum $2 \mathrm{D}$ spectroscopy, Opt. Lett. 41, 2954 (2016).

[13] X. Dai, M. Richter, H. Li, A. D. Bristow, C. Falvo, S. Mukamel, and S.T. Cundiff, Two-Dimensional DoubleQuantum Spectra Reveal Collective Resonances in an Atomic Vapor, Phys. Rev. Lett. 108, 193201 (2012).

[14] A. M. Reilly and A. Tkatchenko, Role of Dispersion Interactions in the Polymorphism and Entropic Stabilization of the Aspirin Crystal, Phys. Rev. Lett. 113, 055701 (2014).

[15] N. Laman, S. S. Harsha, and D. Grischkowsky, Narrow-line waveguide terahertz time-domain spectroscopy of aspirin and aspirin precursors, Appl. Spectrosc. 62, 319 (2008).

[16] C. C. Wilson, Interesting proton behavior in molecular structures. Variable temperature neutron diffraction and $a b$ initio study of acetylsalicylic acid: Characterizing librational motions and comparing protons in different hydrogen bonding potentials, New J. Chem. 26, 1733 (2002).

[17] W. Kuehn, K. Reimann, M. Woerner, T. Elsaesser, and R. Hey, Two-dimensional terahertz correlation spectra of electronic excitations in semiconductor quantum wells, J. Phys. Chem. B 115, 5448 (2011).

[18] M. Woerner, W. Kuehn, P. Bowlan, K. Reimann, and T. Elsaesser, Ultrafast two-dimensional terahertz spectroscopy of elementary excitations in solids, New J. Phys. 15, 025039 (2013).

[19] G. Folpini, D. Morrill, C. Somma, K. Reimann, M. Woerner, T. Elsaesser, and K. Biermann, Nonresonant coherent control-intersubband excitations manipulated by a nonresonant terahertz pulse, Phys. Rev. B 92, 085306 (2015).

[20] C. Somma, G. Folpini, K. Reimann, M. Woerner, and T. Elsaesser, Two-Phonon Quantum Coherences in Indium Antimonide Studied by Nonlinear Two-Dimensional Terahertz Spectroscopy, Phys. Rev. Lett. 116, 177401 (2016).

[21] C. Somma, G. Folpini, K. Reimann, M. Woerner, and T. Elsaesser, Phase-resolved two-dimensional terahertz spectroscopy including off-resonant interactions beyond the $\chi^{(3)}$ limit, J. Chem. Phys. 144, 184202 (2016).

[22] Q. Wu and X.-C. Zhang, Free-space electro-optic sampling of terahertz beams, Appl. Phys. Lett. 67, 3523 (1995).

[23] Q. Wu and X.-C. Zhang, Free-space electro-optics sampling of midinfrared pulses, Appl. Phys. Lett. 71, 1285 (1997).

[24] See Supplemental Material at http://link.aps.org/ supplemental/10.1103/PhysRevLett.119.097404, which includes Refs. [25-31] and contains additional details on experimental effects and on the theoretical calculations.

[25] R. Ruppin, Surface effects on optical phonons and on phonon-plasmon modes, Surf. Sci. 34, 20 (1973).

[26] T. Tyborski, R. Costard, M. Woerner, and T. Elsaesser, Ultrafast vibrational dynamics of $\mathrm{BH}_{4}^{-}$ions in liquid and 
crystalline environments, J. Chem. Phys. 141, 034506 (2014).

[27] V. Blum, R. Gehrke, F. Hanke, P. Havu, V. Havu, X. Ren, K. Reuter, and M. Scheffler, $A b$ initio molecular simulations with numeric atom-centered orbitals, Comput. Phys. Commun. 180, 2175 (2009).

[28] A. Marek, V. Blum, R. Johanni, V. Havu, B. Lang, T. Auckenthaler, A. Heinecke, H.-J. Bungartz, and H. Lederer, The ELPA library: Scalable parallel eigenvalue solutions for electronic structure theory and computational science, J. Phys. Condens. Matter 26, 213201 (2014).

[29] T. Auckenthaler, V. Blum, H.-J. Bungartz, T. Huckle, R. Johanni, L. Krämer, B. Lang, H. Lederer, and P. Willems, Parallel solution of partial symmetric eigenvalue problems from electronic structure calculations, Parallel Comput. 37, 783 (2011).

[30] V. Havu, V. Blum, P. Havu, and M. Scheffler, Efficient O(N) integration for all-electron electronic structure calculation using numeric basis functions, J. Comput. Phys. 228, 8367 (2009).

[31] A. Togo and I. Tanaka, First-principles phonon calculations in materials science, Scr. Mater. 108, 1 (2015).

[32] T. Siebert, B. Guchhait, Y. Liu, B. P. Fingerhut, and T. Elsaesser, Range, magnitude, and ultrafast dynamics of electric fields at the hydrated DNA surface, J. Phys. Chem. Lett. 7, 3131 (2016).

[33] J. P. Perdew, K. Burke, and M. Ernzerhof, Generalized Gradient Approximation Made Simple, Phys. Rev. Lett. 77, 3865 (1996); Erratum, Phys. Rev. Lett. 78, 1396(E) (1997).

[34] A. Tkatchenko, R. A. DiStasio, Jr., R. Car, and M. Scheffler, Accurate and Efficient Method for Many-Body van der Waals interactions, Phys. Rev. Lett. 108, 236402 (2012).

[35] A. Ambrosetti, A. M. Reilly, R. A. DiStasio Jr., and A. Tkatchenko, Long-range correlation energy calculated from coupled atomic response functions, J. Chem. Phys. 140, 18A508 (2014). 